\title{
EVALUATION OF THE THICKNESS AND DEPTH OF PENETRATION OF ICON INTO THE ARTIFICIAL ENAMEL WHITE SPOT LESION
}

\author{
Abdallah. A. A. Abd Alhady* and Hamed Ibrahim Mohamed*
}

\begin{abstract}
This research was designed to compare the thickness and the depth of penetration of two resin-based materials (Icon and one-step self-etch adhesive (Single Bond Universal)) in artificial white spot lesion (WSL) created on caries-free molar teeth, without and with surface treatment prior application of the resin-based materials at different storage time (one day, one week and one month). The 126 samples were randomly divided into three equal main groups $(n=42)$ according to the resinous material (M); Group 1 (M1) was considerate to be control group. Group 2 (M2) was restored with Icon. Group 3 (M3) was restored with Single Bond Universal. Each group was divided into two equal subgroups $(n=21)$ according to the surface treatment $(\mathrm{W})$; Where the first subgroup (W1) was subjected to surface treatment while the second subgroup (W2) was without surface treatment. The samples were further divided into three divisions $(n=7)$ according to the storage time (S). Division 1 (S1) was stored for one day. Division 2 (S2) was stored for one week. Division 3 (S3) was stored for one month. Each resin-based material was applied after creation of white spot lesion, according to the manufacture instruction. Buccal and lingual surface of each sample was used to measure the thickness of the resin-based materials using Optical profilometer while confocal laser scanning microscopy (CLSM) was used to measure the depth of penetration. The result of this study reveals that: the surface treatment prior application of Icon group improve the degree of penetration depth to get the highest value compared with the other groups while the thickness of the untreated surface prior application of Icon groups gives the thickest value compared with the thickness of the other groups. Conclusion; Icon is the best choice for treatment of (WSLs).
\end{abstract}

\section{INTRODUCTION}

Orthodontic corrections are mostly achieved with fixed orthodontic appliances. One of the negative side-effects is the formation of white spot lesions (WSL) and incipient caries. Prevention of (WSL) during fixed appliances orthodontic treatment is still a challenge in today's orthodontic treatment: There is evidence that neglecting oral hygiene during orthodontic treatment with fixed appliances can cause WSL formation within weeks. ${ }^{(1)}$ White spot lesions (WSLs) are early signs of demineralization

\footnotetext{
* Lecture in Operative Department, Faculty of Dental Medicine, Al-Azhar University (Cairo).
} 
under intact enamel, which may or may not lead to the development of caries. The another causes of (WSLs) may include plaque accumulation particularly along the cervical margins of teeth, inadequate home oral care, and/or consumption of diets rich in sugar those that frequently lower the intraoral $\mathrm{PH} .{ }^{(2)}$ The recently introduced alternative therapy is the use of resin-based materials over the dental tissue which completely fills pores within the tooth, replacing lost tooth structure and stopping caries progression., caries infiltration technique; Icon has been used as a promising therapeutic method for non-cavitated lesions. The enamel pores system is filled or reinforced with low-viscosity a light-curable resin. In contrast to conventional sealants, in which the material adheres to the enamel surface, resin infiltration penetrates into the porous lesion body of enamel's initial carious lesions using a special low-viscosity resin that blocks the diffusion of acids into the lesion, so it blocks the demineralizing effects of cariogenic acids, thereby slowing or arresting the progression of caries. ${ }^{(3,4)}$ Self-etch adhesives contain high concentration of solvents which must be eliminated after complete their function because the residual solvent lead to deterioration of the adhesive interface between tooth structure and composite resin by interfering with TABLE (1): Materials used in the study: resin polymerization. Complete solvent elimination by air drying difficult to achieve, consequently, some residual solvent remains trapped in the adhesive. (5) Also, pretreatment of the (WSLs) surface with phosphoric acid etch may show a higher result of penetration coefficient as it allows for more surface area to be coated by resin-based material. As the main idea of self-adhesive system is to reduce dentine sensitivity and as long as the (WSLs) are confined in enamel only, so surface treatment may add value to its penetration depth into (WSL) without postoperative sensitivity ${ }^{(8)}$. Confocal laser scanning microscopy (CLSM) where used to determine the amount of resin-based material penetrated inside the (WSLs). Thus, the success of infiltration technique, depends on the efficacy of this low viscosity resin to penetrate up to the depth of the (WSL) and not just mask the lesions. ${ }^{(9)}$.Although clinical studies have been done earlier. They focused mainly on the clinical success and outcome of the resin. Depth of resin penetration could be a key determining factor for the creation of a diffusion barrier and the success of infiltration. ${ }^{(8)}$

\section{MATERIALS \& METHODS}

All the materials compositions are listed according to the manufacturers' profile

\begin{tabular}{|l|l|l|l|l|}
\hline & Brand name & Composition & Manufacturer & Lot Number \\
\hline 1- & $\begin{array}{l}\text { Scotchbond } \\
\text { Universal Etchant }\end{array}$ & $\begin{array}{l}32 \% \text { phosphoric acid by weight and has a } \\
\text { pH of approximately 0.1. fumed silica and a } \\
\text { water soluble polymer }\end{array}$ & $\begin{array}{l}\text { 3M ESPE Dental Products,2510, } \\
\text { Conway Avenue, St. Paul, MN } \\
\text { 55144-1000 USA } \\
\text { www.3MESPE.com }\end{array}$ & 6520011690261 \\
\hline 2- & Icon & $\begin{array}{l}\text { Icon-Etch: Hydrochloric acid, pyrogenic } \\
\text { silicic acid, surface-active substances } \\
\text { Icon-Dry: 99\% ethanol } \\
\text { Icon-Infiltrant: Methacrylate-based resin } \\
\text { matrix, initiators, additives }\end{array}$ & $\begin{array}{l}\text { DMG Chemisch- } \\
\text { Pharmazeutische Fabrik,GmbH } \\
\text { Elbgaustraße 248 22547 } \\
\text { Hamburg www.dmg-america. } \\
\text { com }\end{array}$ & 220401 \\
\hline 3- & $\begin{array}{l}\text { MDP phosphate monomer Dimethacrylate } \\
\text { resins, HEMA, Vitrebond Copolymer, } \\
\text { filler, initiators, silane, and ethanol water. }\end{array}$ & $\begin{array}{l}\text { 3M ESPE St.Paul, MN,USA } \\
\text { www.3m.com }\end{array}$ & 606115 \\
\hline
\end{tabular}




\section{Methods:}

\section{Selection of teeth and grouping of specimens:}

A total number of 63 human posterior teeth, free from caries extracted for a pathological reason were collected to be used in this study. 126 samples of buccal and lingual surfaces were obtained from these teeth. The samples were randomly divided into three equal main groups $(n=42)$ according to the resinous material (M). Group 1 (M1) was considerate to be control group. Group 2 (M2) was restored with Icon. Group 3 (M3) was restored with Single Bond Universal. Each group was divided into two equal subgroups $(n=21)$ according to the surface treatment (W). Where the first subgroup (W1) was subjected to surface treatment while the second subgroup (W2) was without surface treatment. The samples were further divided into three divisions $(\mathrm{n}=7)$ according to the storage time $(\mathrm{S})$. Division 1 (S1) was stored for one day. Division 2 (S2) was stored for one week. Division 3 (S3) was stored for one month.

\section{Preparation of the specimens;}

126 samples were subjected to a short-term acidic exposure by application of phosphoric acid $\mathrm{H} 3 \mathrm{PO} 437 \%$ to the buccal and lingual surfaces for one minute to create artificial (WSLs). ${ }^{(3)}$ Half of these samples were surface treated again with phosphoric acid H3PO4 37\% for thirty second and rinse with water for five second then air dried with oil-free air spray while the other samples not treated. Then the resin based material applied, according to manufacture instructions and immersed in artificial saliva which daily changed.

\section{I- Measurements of resin-based materials thickness}

Acid etch (surface treatment) was applied on the buccal or lingual surface of half of the samples
(63) as discuss previously after creation of artificial (WSLs), then mark was made on the model surface corresponding to the etched surface either its buccal or lingual to be easy define between etched (treated surface W1) and unetched (untreated surface W2). Profilometric analysis was performed at the treated and untreated surfaces to be a baseline measurement.

\section{Application of the resin-based materials:}

Each resin-based material was applied according to the manufacture instruction as the following steps:

\section{Icon application procedure:}

Icon-Etch (hydrochloric acid) was applied and allowed to sit for two min then rinsed off with air water spray for at least thirty second, then samples were dried gently with oil free air. Icon-Dry was applied to the lesion site and left for thirty second, follows by gently air drying. Icon-Infiltrant was applied to the etched surface and allowed to sit for three min, then light-cured for forty sec. using Elipar Light cure unit.

\section{Single Bond Universal application procedure:}

Apply Single Bond Universal Primer to the enamel surface using the disposable applicator brush. Scrub the surface with brushing motion for 20 seconds. Then air thin for 5 seconds with medium air pressure shake the adhesive bottle briefly then, apply to the enamel surface with light brushing motion for 15 seconds. Again, air thin for 5 seconds. Then light cure using Elipar Light cure unit for 10 seconds.

\section{Resin penetration depth measurement using (CLSM)}

Two randomly teeth selected from each (W1) and (W2) groups. Then picture captured for the samples by (CLSM) were assessed and the penetration depth of the resin-based materials were measured and compared each result with each other group. 
TABLE (2): Mean and standard deviation of the resin-based materials thickness in $(\mu \mathrm{m})$ along the storage time.

\begin{tabular}{|c|c|c|c|c|c|}
\hline \multirow{2}{*}{$\begin{array}{c}\text { Storage } \\
\text { time. }\end{array}$} & $\begin{array}{c}\text { Surface } \\
\text { Treatment }\end{array}$ & $\begin{array}{c}\text { Control } \\
\text { group } \\
\text { M1 }\end{array}$ & $\begin{array}{c}\text { Icon } \\
\text { group } \\
\text { M2 }\end{array}$ & $\begin{array}{c}\text { Single } \\
\text { Bond } \\
\text { universal }\end{array}$ & P* \\
\hline \multirow{2}{*}{ S1 } & W1 & $0.23876 \pm 0.1231$ & $0.25376 \pm 0.1124$ & $0.24676 \pm 0.1180$ & 0.984 \\
\cline { 2 - 6 } & W2 & $0.24691 \pm 0.1012$ & $0.26906 \pm 0.1178$ & $0.25167 \pm 0.1080$ & 0.950 \\
\hline \multirow{2}{*}{ S2 } & W1 & $0.22728 \pm 0.0976$ & $0.24570 \pm 0.1035$ & $0.23652 \pm 0.1107$ & 0.974 \\
\cline { 2 - 7 } & W2 & $0.24554 \pm 0.1206$ & $0.25299 \pm 0.0966$ & $0.24844 \pm 0.1117$ & 0.998 \\
\hline \multirow{2}{*}{ S3 } & W1 & $0.21547 \pm 0.0909$ & $0.24299 \pm 0.1315$ & $0.23213 \pm 0.0946$ & 0.908 \\
\cline { 2 - 7 } & W2 & $0.23317 \pm 0.0935$ & $0.25124 \pm 0.0994$ & $0.24748 \pm 0.1126$ & 0.970 \\
\hline \multicolumn{2}{|c|}{ Average thickness } & $0.23452 \pm 0.1055$ & $0.25262 \pm 0.1102$ & $0.24182 \pm 0.1093$ & 0.719 \\
\hline
\end{tabular}

\section{Results of the profilometric analysis:}

\section{Comparison between the average thicknesses of the resin-based materials.}

The results of this study Table (2) and Figure (1), record that the highest thickness recorded in case of Icon group $(0.25262 \pm 0.1102)$, this followed by the Single Bond Universal group (0.24182 \pm 0.1093$)$, The difference between different average thickness was statistically not significant.

\section{Control group (M1):}

It was found that the highest average thickness $(0.24691 \pm 0.1012)$ recorded in day one without

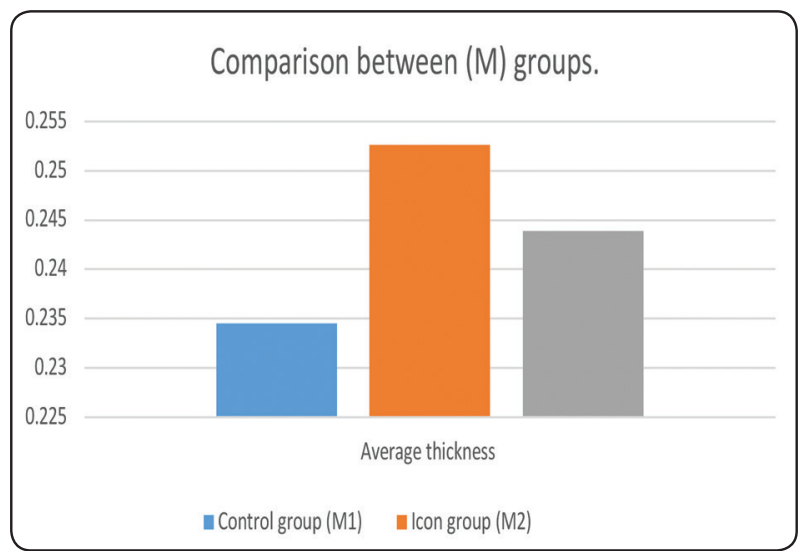

Fig. (1): Comparison between the thicknesses of different resinbased materials. surface treatment group (W2S1) while, the lowest average thickness $(0.21547 \pm 0.0909)$ recorded after one-month with surface treated group (W1S3). The difference between different average thickness was statistically not significant $(\mathrm{P}>0.05)$

\section{Icon group (M2):}

It was found that the highest average thickness $(0.26906 \pm 0.1178)$ recorded in day one without surface treatment group (W2S1) while, the lowest average thickness $(0.24299 \pm 0.1315)$ recorded after one-month with surface treated group (W1S3). The difference between different average thickness was statistically not significant $(\mathrm{P}>0.05)$ as Single Bond universal group (M3):

It was found that the highest average thickness $(0.25157 \pm 0.1080)$ recorded in day one without surface treatment group (W2S1) while, the lowest average thickness $(0.23113 \pm 0.0946)$ recorded after one-month with surface treated group (W1S3). The difference between different average thickness was statistically not significant $(\mathrm{P}>0.05)$.

\section{Surface treatment effect on the thickness of resin- based materials:}

It was found that the (W2) $(0.248249 \pm 0.1846)$ groups recorded the highest average thickness 
while, the (W1) groups recorded the lowest average thickness $(0.235748 \pm 0.1069)$. The difference between different average thickness was statistically not significant $(\mathrm{P}>0.05)$

Comparison of the surface treatment (W1) effect on each resin-based materials groups:

It was found that the Icon group at day one (S1M2) recorded the highest average thickness $(0.25376 \pm 0.1124)$ followed by single bond universal (S1M3) group at day one $(0.24566 \pm 0.1180)$, the difference between different average thickness was statistically not significant $(\mathrm{P}>0.05)$

\section{Comparison of the thickness of each resin-based materials groups without surface treatment (W2)}

It was found that the Icon group at day one (S1M2) recorded the highest average thickness $(0.26906 \pm 0.1178)$ followed by single bond universal (S2M2) group at day one $(0.25299 \pm 0.0966)$, the difference between different average thickness was statistically not significant $(\mathrm{P}>0.05)$

\section{Storage time effect on the thickness of resin-based materials}

It was found that the day one (S1) subgroups recorded the highest average thickness, followed by the one-week (S2) subgroups while, the onemonth (S3) subgroups recorded the lowest average thickness. The difference between different average thickness was statistically not significant $(\mathrm{P}>0.05)$.

Comparison of the storage time effect on the thickness of resin-based materials groups:

It was found that the highest thickness recorded of the resin-based materials at day one $(0.24932 \pm 0.1136)$, followed by one week $(0.24503 \pm 0.1073)$ and lowest after one month $(0.23407 \pm 0.2161)$. With no significant difference $(\mathrm{P}>0.05)$.
Comparison between the Effect of the day one (S1) storage time on the Thickness of (W2) and (W1) groups in each (M) groups:

It was found that the Icon groups (W2M2) groups recorded the highest average thickness, followed by the Icon groups (W1M2), and followed by single bond universal group (W2M3), the difference between different average thicknesses was statistically not significant.

Comparison between the Effect of the one-week (S2) storage time on the Thickness of (W2) and (W1) groups in each (M) groups :

It was found that after storage for one week, the Icon groups (W2M2) groups recorded the highest average thickness, followed by single bond universal group (W2M3), while, the difference between different average thicknesses was statistically not significant.

Comparison between the Effect of the one-month (S3) storage time on the Thickness of (W2) and (W1) groups in each (M) groups:

It was found that after storage for one month, the Icon groups (W2M2) groups recorded the highest average thickness, followed by single bond universal group (W2M3), the difference between different average thicknesses was statistically not significant

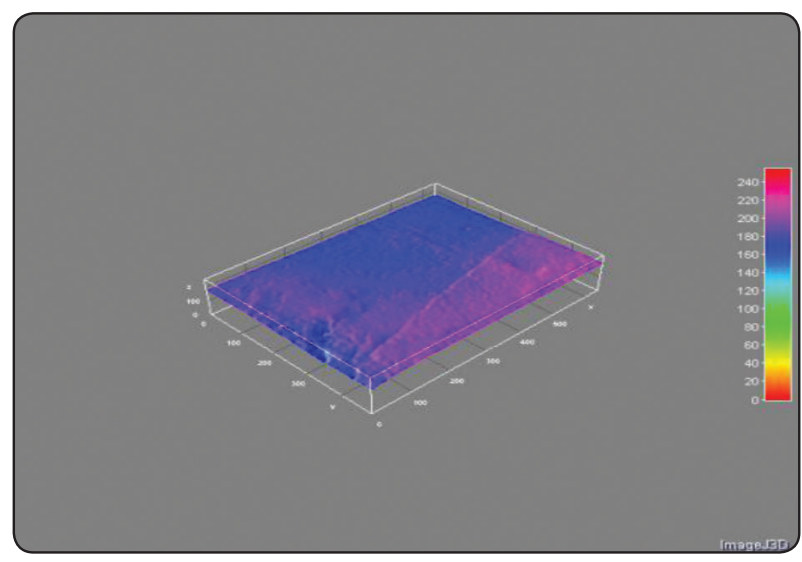

Fig. (2): Optical scan of the sample after application of resinbased material. 


\section{Profilometer photo scanning results:}

\section{Results of the (CLSM):}

It was found that the highest penetration depth was (mean $\pm \mathrm{SD} ; 7.15 \pm 3.551)$ recorded in groups treated with Icon (M2) after being subjected to surface treatment (W1), and the lowest penetration was (mean $\pm \mathrm{SD} ; 1.65 \pm 0.709$ ) observed in groups treated with single bond universal (M2) without surface treatment (W2). The ANOVA test revealed that there is statistically significant difference in the penetration depth of tested materials $\mathrm{p}<0.05$.

\section{Effect of surface treatment on the penetration depth of each (M) groups:}

It was found that the (W1) groups recorded the highest penetration depth values (4.23(2.01)) Figure $(32,34 \& 36)$ while, the (W2) groups recorded the lowest penetration depth values $(2.02(0.948)$, the difference between different average thickness was statistically significant $(\mathrm{P}>0.05)$

\section{(CLSM) photo result:}

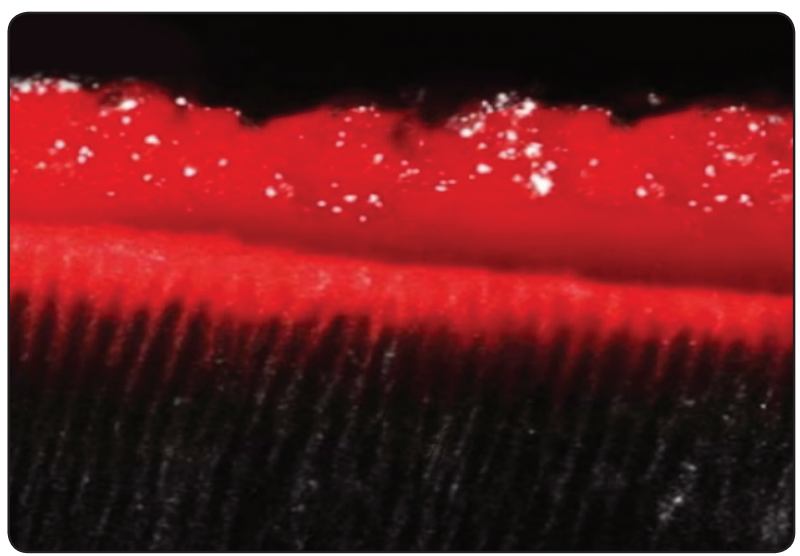

Fig. (3): Icon penetration depth with surface treatment

\section{DISCUSSION}

\section{Comparison between the different resin-based materials}

Result of the current study revealed that: Icon group recorded the highest vertical-height mean value which means the lowest surface loss. This may be due to: Icon prevents any further progression of the enamel (WSLs) by occluding the micro porosities inside the lesion body by infiltration that have been optimized for rapid penetration and improving the surface height and hardness of the treated teeth.

This is in agreement with Belli et al, in (2011) who found that: The vertical surface loss values of the (WSLs) treated with the resin infiltration showed the same result of the original enamel, indicating that this material might be suitable for the treatment of enamel WSL. ${ }^{(6)}$ This is also in agreement with D Rios et al, in (2015) who found that: The Icon was able to protect the enamel. And even after the erosive challenge, the thichness of the Icon that covered the enamel surface was nearly the same, regardless of enamel conditioning. ${ }^{(4)}$ This is also in agreement with Senestraro et al, in (2013) who found that: Resin infiltration significantly improved the clinical appearance of WSLs, with stable results seen eight weeks after treatment. Practically resin infiltration, a minimally invasive restorative treatment, was shown to be effective for WSLs that formed during orthodontic treatment. ${ }^{(8)}$

This disagrees with Wolfgang $\mathrm{H}$ et al, in (2014) who found that: Resin infiltration has some limitations of its technique that are surface conditioning, porosity of the lesion and the extreme hydrophobicity of the resin. This controversy may be due to the using of different manufacture lot or even storage media that couldn't match manufacture storage instruction. Perhaps the test and research that have been done on the Icon gives approximately the same result without mention that limitations. ${ }^{(9)}$ Also disagreement with Taher et al, in (2012) who concluded that the Icon surface pretreatment sealed the enamel porosities in the infiltrated enamel but not improves the surface hardness compared to sound enamel. ${ }^{(10)}$

However, there were no significant difference single bond universal group and Icon group in the surface loss, this may be due to: The chemical 
composition and stability of the material itself. That could lead for some sort of surface loss from the material. This agrees with Rahiotis C et al, (2011) who found that: when the Icon versus the two-step adhesive system applied over the caries lesion was compared with the original enamel, non-significant differences in vertical surface loss were measured. However, the Icon material showed surface and morphological aspects that pointed to improved surface stability and infiltration quality Thus, a thicker layer of Icon might be beneficial for surface loss resistance. ${ }^{(7)}$

\section{Comparison of the surface treatment (W1) effect on each resin-based materials groups:}

Result of the current study revealed that: the untreated surface groups (W2) recorded the highest vertical-height mean value which means the lowest surface loss. This may be due to: The excess loss of tooth structure during surface treatment, which in turn increase the depth of the lesion and of course will need for more material to be restored. As long as each material has it stander thickness so when restoring (WSLs) it will occlude the whole vertical loss of the lesion. So by scanning the restored surface of the lesion with optical profilometer. More surface highest will be shown on the surface of the lesion in comparison with surface treated groups (W1). However, there is no significant difference between the two results. This is in agreement with Oliveira et al, in (2015) who found that: The thickest layer resulted from the application of resin infiltrant; however, there was no significant difference compared to the self-etching adhesive and pit \& fissure sealant with or without surface treatment. When enamel etching was performed. After application of the conventional adhesive with previous enamel etching, negative values, which represent the absence of material over enamel and even enamel loss, were observed. This group showed no significant differences between the same material and the self-etching adhesive without conditioning, since both materials showed a very thin layer of material. ${ }^{(4)}$

This also in agreement with Tereza et al, in (2016) who found that: Different resin-based material thickness and enamel wear were assessed using profilometry. Resulted in the formation of a layer of material over enamel, being similar effective in inhibiting erosion progression. So resinbased materials are able to protect enamel against erosion only when they are present over enamel, as a physical barrier. ${ }^{(11)}$

\section{Storage time effect on the thickness of resin- based materials:}

Result of the current study revealed that: The day one (S1) subgroups recorded the highest average thickness while, the one-month (S3) subgroups recorded the lowest average thickness. This is in agreement with HM Hono'rio et al, in (2015) who found that: the Icon material showed surface and morphological aspects that pointed to improved surface stability and infiltration quality. Thus, a thicker layer of infiltrant might be beneficial for surface loss resistance. ${ }^{(4)}$ Also in this study: the control group show the lowest vertical height values at the end of the month which mean that the (WSL) continues in progressions and cause more loss of tooth structure as it left untreated. This is in agreement with Bishara et al, in (2008) who found that: If (WSLs) are left untreated, they may progress to produce carious cavitations, and may also present esthetic problems. ${ }^{(12)}$

\section{Comparison of Penetration depth of the resin- based materials:}

Result of the current study (Table 20) revealed that: The highest penetration depth was recorded in Icon group with surface treatment (WM2). This may be due to: The Icon is basically composed of the monomer triethylene glycol dimethacrylate (TEGDMA) and resinous sealant consists of TEGDMA and bisphenol glycidyl methacrylate 
(BisGMA) monomers. The TEGDMA based materials show greater penetration due to their low viscosity, high degree of conversion, and high penetration coefficient. This is in agreement with Ionta et al, in (2016) who found that: The Icon showed greater depth and homogeneity of penetration than the other materials, followed by the resinous sealant. ${ }^{(12)}$

However, the (single bond universal) group recorded the mean value of the depth of penetration less than Icon group but higher than the control group. This may be due to: the presence of the hydroxyethyl methacrylate (HEMA) which has less penetration ability in comparison with Icon group. This is in agreement with Ionta et al, in (2016) who found that: It's contain and ethanol in its composition, this combination might results on insufficient polymerization (rubber or liquid consistency) This characteristic may have hampered the penetration and adhesion Despite the high penetration coefficient of that material. ${ }^{(9)}$

\section{Effect of the surface treatment on the penetra- tion depth of each group (M)}

Result of the current study revealed that: All groups that have been treated with surface conditioning reveals the highest mean value of penetration. This may be due to: The more surface area and pores plus the dissolution of the prismatic layer of the enamel. This in agreement with Ionta et al, in (2016) who found that: The etching with phosphoric acid improves the mechanical retention of dental materials by promoting the dissolution of prismatic and inter-prismatic enamel and creating irregularities in the enamel "prisms" in which the resin-based material can flow. ${ }^{(12)}$ This also, in agreement with Pashley et al,(2011) who found that: Etch-and-rinse adhesives produce higher resin-dentin bonds that are more durable than most 1 and 2-step adhesives. ${ }^{(13)}$ This also, in agreement with Hanabusa et al, in (2012) who found that: Prior phosphoric-acid etching significantly increased the bonding effectiveness of the resin-based material to enamel. The phosphoric-acid etching definitely improved bonding of the one-step self-etch adhesive to enamel. ${ }^{(14)}$

\section{CONCLUSIONS}

1- Icon is the best choice for treatment of (WSLs)

2- Surface treatment has positive effect on the penetration depth of the resin-based materials, while it's have a negative effect on the resinbased materials thickness.

3- Storage time has a negative effect on the thickness of the resin-based materials.

\section{REFERENCES}

1. Knösel M, Vogel R, Sandoval P. Infiltration of WhiteSpot-Lesions and developmental enamel defects. nRev. Clin. Periodoncia Implantol. Rehabil. Oral 2017; 10(2); 101-6.

2. Shivanna V, Shivakumar B. Novel treatment of white spot lesions: A report of two cases. J Conserv Dent. 2011 Oct; 14(4):423-6

3. Alhamadi W, Osman E, Saleh F, AL-Mayali A, Alhumadi A, Alrufaye Z. Effect of Resin Infiltrant Pretreatment on Shear Bond Strength of Metal Orthodontic Brackets in Vitro Study Int J Dent \& Oral Heal.2018; 4:7, 101-6.

4. Gugnani N, Pandit I, Gupta M, Josan R. Caries infiltration of noncavitated white spot lesions: A novel approach for immediate esthetic improvement. Contemp Clin Dent. 2012; 3(6):199.

5. Itthagarun A, Tay FR, Pashley DH, Wefel JS, GarcíaGodoy F, Wei SHY. Single-step, self-etch adhesives behave as permeable membranes after polymerization. Part III. Evidence from fluid conductance and artificial caries inhibition. Am J Dent. 2004 Dec;17(6):394-400.

6. Oliveira G, Boteon A, Ionta F, Moretto M, Honório H, Wang L, et al. In Vitro Effects of Resin Infiltration on Enamel Erosion Inhibition. Oper Dent. 2015;40(5):492502 .

7. Jonkman J, Brown CM. Any way you slice it-A comparison of confocal microscopy techniques. J Biomol Tech. 2015;26(2):54-65. 
8. Paris S, Meyer-Lueckel H, Cölfen H, Kielbassa AM. Penetration coefficients of commercially available and experimental composites intended to infiltrate enamel carious lesions. Dent Mater. 2007 Jun;23(6):742-8.

9. Belli R, Rahiotis C, Schubert EW, Baratieri LN, Petschelt A, Lohbauer U. Wear and morphology of infiltrated white spot lesions. J Dent. 2011;39(5):376-85.

10. Senestraro S V., Crowe JJ, Wang M, Vo A, Huang G, Ferracane $\mathrm{J}$, et al. Minimally invasive resin infiltration of arrested white-spot lesions: A randomized clinical trial. J Am Dent Assoc. 2013;144(9):997-1005.

11. H. Arnold W, Bachstaedter L, Benz K, A. Naumova E. Resin Infiltration into Differentially Extended Experimental Carious Lesions. Open Dent J. 2014;8(1):251-6.

12. Elhiny OA, Elattar HS, Salem GA. The influence of resin infiltration system on sound enamel microhardness and shear-bond strength of orthodontic bands: An in-vitro study. Der Pharma Chem. 2016;8(18):100-6.

13. Tereza GPG, De Oliveira GC, De Andrade Moreira Machado MA, De Oliveira TM, Da Silva TC, Rios D. Influence of removing excess of resin-based materials applied to eroded enamel on the resistance to erosive challenge. $\mathrm{J}$ Dent. 2016 Apr; 47(1):49-54.

14. Bishara SE, Ostby AW. White Spot Lesions: Formation, Prevention, and Treatment. Semin Orthod. 2008;14(3): 174-82.

15. Pashley DH, Tay FR, Breschi L, Tjäderhane L, Carvalho RM, Carrilho M, et al. State of the art etch-and-rinse adhesives. Dent Mater. 2011;27(1):1-16.

16. Hanabusa M, Mine A, Kuboki T, Momoi Y, Van Ende A, Van Meerbeek B, et al. Bonding effectiveness of a new "multi-mode" adhesive to enamel and dentine. J Dent. 2012 Jun;40(6):475-84. 